ISSN: 0213-2087 eISSN: 2444-7080

DOI: http://dx.doi.org/10.14201/shhc2018362534

\title{
ALGUNOS ELEMENTOS BÁSICOS DE LA «CULTURA DE LOS DERECHOS» ¿POR QUÉ LOS DERECHOS HUMANOS?
}

\section{Some basic elements of the "Culture of Rights". Why Human Rights?}

Javier de LUCAS

Universitat de València

lucasfra@uv.es

Recepción: 27/04/2018 Revisión: 23/05/2018 Aceptación: 25/06/2018

RESUMEN: El autor propone una reflexión sobre dos elementos básicos de la "cultura de los derechos", la idea de lucha por los derechos, tras las tesis del jurista alemán Jhering, y la reformulación de la noción de universalidad como universabilidad, desde tres consideraciones: la idea de egalibertad, el carácter inacabado e inacabable del proceso de reconocimiento y garantía de los derechos y la radical dimensión política de los derechos humanos como condición de legitimidad.

Palabras clave: derechos humanos; egalibertad; lucha por el Derecho; legitimidad.

ABSTRACT: The author proposes a reflection on two basic elements of the "culture of rights", the idea of struggle for rights, following the thesis of the german jurist Jhering, and the reformulation of the notion of universality, from three considerations: idea of <equaliberty> (Balibar), the unfinished and endless character of the process of recognition and guarantee of rights and the radical political dimension of human rights as a condition of legitimacy.

Key words: human rights; equaliberty; Struggle for Law; legitimacy. 
JAVIER DE LUCAS

ALGUNOS ELEMENTOS BÁSICOS DE LA «CULTURA DE LOS DERECHOS» ¿POR QUÉ LOS DERECHOS HUMANOS?

\section{INTRODUCCIÓN}

Para muchos de nosotros, este de 2018 es, sobre todo, en el ámbito de los derechos humanos, el año del 70 aniversario de la Declaración universal de derechos, el texto más traducido y de mayor difusión contemporánea. Incorporado a casi todas las Constituciones y Cartas o Declaraciones de derechos nacionales y regionales, su importancia es difícil de exagerar. Y, sin embargo, estamos lejos aún de alcanzar lo que podríamos denominar una "cultura universal y básica de los derechos".

La primera dificultad para alcanzar ese objetivo reside, por supuesto, en la ausencia de una voluntad política de compromiso con esa tarea de consolidación de cultura de derechos que es condición sine qua non, a mi juicio, del núcleo de legitimidad democrática exigible hoy, porque lo es también de la concreción histórica, hoy, de la exigencia mínima de justicia, la primacía del reconocimiento y garantía de los derechos humanos. Junto a ello, habría que hacer frente a la objeción del plural: ¿cómo hablar de una cultura de derechos sin despreciar o contradecir la constatable existencia de la pluralidad cultural, una dimensión que habría sido ignorada precisamente en la gestación de la Declaración universal, en 1948?

En lo que sigue, trato de proponer de forma sencilla dos elementos básicos para apuntalar esa cultura de los derechos. En primer lugar, la noción de <lucha por los derechos> que, aunque tiene antecedentes tan notables como los que hallamos en algunos de los fragmentos de Heráclito, es formulada sobre todo por un excepcional jurista del siglo xix, el alemán Rudolf von Jhering. La segunda, es la aspiración a la universalidad y digo aspiración porque más que de universalidad de los derechos humanos me gusta hablar de universabilidad de los mismos, para poder formular mejor esta característica y hacerla conjugable con el respeto al pluralismo de concepciones de los derechos, que no es solo, aunque esto sea muy importante, el respeto al pluralismo cultural, una tesis que no siempre es bien formulada y, menos aún, entendida. De esa formulación de la universalidad se desprenden, como propondré, tres consideraciones elementales que han de ser tenidas en cuenta.

\section{LA NOCIÓN DE «LUCHA POR LOS DERECHOS», NÚCLEO DE LA IDEA DE DERECHOS HUMANOS}

La noción misma de Derecho, como advirtiera Jhering, es inseparable de la idea de lucha por el Derecho, que lo es, a su vez, de la lucha por el reconocimiento de los derechos humanos. Bien puede decirse que su lema es una de las tesis más acertadas sobre el sentido del Derecho como producto cultural: «todo Derecho en el mundo tuvo que ser adquirido mediante la lucha». De esta manera, además, el origen último del Derecho tendría una motivación ética, es decir, reconoce la existencia de un impulso moral en cada uno de los seres humanos -el sentimiento de lo justo, que es el sentimiento del Derecho, que subyace a cada uno de nosotros. Pero también es posible encontrar en ella una profunda significación política, de 
carácter democrático: somos todos nosotros quienes creamos el reconocimiento de esos derechos; todos debemos estar implicados en ese objetivo, que nunca estará seguro, nunca se podrá dar por definitivamente alcanzado, si no tiene ese respaldo.

En efecto, el reconocimiento de los derechos humanos (su garantia, también) no puede desvincularse de procesos de luchas sociales, en su mayoría inacabadas e inacabables que en sus inicios fueron y son presentadas como proyectos utópicos, incluso, desmentidos por los hechos. Por eso muchas veces se asegura que este objetivo de reconocimiento y garantia de los derechos es una empresa tan noble desesperada. Pero cualquier estudioso de la historia de los derechos sabe que no es así. Que lo que la historia de los derechos nos muestra es que, lejos de la utopia, se trata de un proyecto raonable. En dos sentidos: razonable porque es factible, aunque el proceso sea gradual y a veces lento y aunque esté lejos de ser lineal (si algo nos enseña la historia es que los derechos, ningún derecho, se puede considerar jamás como obtenido definitivamente). Razonable además porque tiene la fuerza incontenible de la razón, o, para ser más precisos, la fuerza de toda idea que alcanza la categoria de Zeitgeist, esto es, una idea a la que le llega su tiempo, como asegurara V. Hugo. Vivimos la fase àlgida, aunque algunos les parezca lo contrario (sobe todo a los pesimistas que tienden a generalitzar los aspectos negativos) de lo que, con Bobbio, podríamos denominar la era, el tiempo de los derechos. Una época que nace con el espíritu mismo de la Ilustración, con su objetivo de emancipación de todos los seres humanos, por más que el proyecto ilustrado admite un concepto restringido de ser humano, que -como he escrito en algun otro lugar- tiene género/sexo, edad, etnia/identidad cultural/, raza y clase. Pero este tiempo de los derechos consiste precisamente en el despliegue de un programa que va desprendiéndose de tales limitacions para alcanzar progresivamente el objetivo de la igual libertad de todos los seres humanos, la universalidad, un objetivo que se despliega a través de las luchas sociales contra toda manifestación de discriminación y de dominación, contra toda forma de exclusión.

Para explicar el arraigo de esta noción de lucha por los derechos, suele apelarse a la presencia universal de lo que se describe como «sentimiento elemental de los jurídico»: ¿habrá alguien que no haya asegurado alguna vez: "No hay derecho!», o "tengo/tenemos derecho a esto!»? ¿Qué sentido tienen esas afirmaciones? ¿Por qué las hacemos? ¿Sirven para algo más que para tratar de expresar nuestra indignación, nuestra protesta, la reivindicación de lo que consideramos nuestro?

Es muy posible que muchos de los que hemos pronunciado afirmaciones semejantes, al hacerlo, teníamos el eco más o menos consciente del famoso preámbulo de la Declaración de independencia de 4 de julio de 1776:

Sostenemos como evidentes por sí mismas dichas verdades: que todos los hombres son creados iguales; que son dotados por su Creador de ciertos derechos inalienables; que entre éstos están la Vida, la Libertad y la búsqueda de la Felicidad. Que para garantizar estos derechos se instituyen entre los hombres los gobiernos, que derivan sus poderes legítimos del consentimiento de los gobernados; que 
cuando quiera que una forma de gobierno se haga destructora de estos principios, el pueblo tiene el derecho a reformarla, o abolirla, e instituir un nuevo gobierno que se funde en dichos principios, y a organizar sus poderes en la forma que a su juicio ofrecerá las mayores probabilidades de alcanzar su seguridad y felicidad. La prudencia, claro está, aconsejará que no se cambie por motivos leves y transitorios gobiernos de antiguo establecidos; y, en efecto, toda la experiencia ha demostrado que la humanidad está más dispuesta a padecer, mientras los males sean tolerables, que a hacerse justicia aboliendo las formas a que está acostumbrada. Pero cuando una larga serie de abusos y usurpaciones, dirigida invariablemente al mismo objetivo, evidencia el designio de someter al pueblo a un despotismo absoluto, es su derecho, es su deber, derrocar ese gobierno y proveer de nuevas salvaguardas para su futura seguridad y su felicidad.

O también de las que encontramos en los cinco primeros artículos que los revolucionarios franceses inscribieron en la Declaración de los derechos del hombre $y$ del ciudadano de 26 de agosto de 1789:

- Artículo 1 Los hombres nacen y permanecen libres e iguales en derechos. Las distinciones sociales sólo pueden fundarse en la utilidad común.

- Artículo 2 La finalidad de cualquier asociación política es la protección de los derechos naturales e imprescriptibles del Hombre. Tales derechos son la libertad, la propiedad, la seguridad y la resistencia a la opresión.

- Artículo 3 El principio de toda Soberanía reside esencialmente en la Nación. Ningún cuerpo ni ningún individuo pueden ejercer autoridad alguna que no emane expresamente de ella.

- Artículo 4 La libertad consiste en poder hacer todo lo que no perjudique a los demás. Por ello, el ejercicio de los derechos naturales de cada hombre tan sólo tiene como límites los que garantizan a los demás Miembros de la Sociedad el goce de estos mismos derechos. Tales límites tan sólo pueden ser determinados por la Ley.

- Artículo 5 La Ley sólo tiene derecho a prohibir los actos perjudiciales para la Sociedad. Nada que no esté prohibido por la Ley puede ser impedido, y nadie puede ser obligado a hacer algo que ésta no ordene.

Es la misma tesis que se encuentra en el Preámbulo de la Declaración universal de los derechos humanos, aprobada por la Asamblea General de la ONU el 10 de diciembre de 1948, y en sus dos primeros artículos:

Artículo 1. Todos los seres humanos nacen libres e iguales en dignidad y derechos y, dotados como están de razón y conciencia, deben comportarse fraternalmente los unos con los otros.

Artículo 2. Toda persona tiene todos los derechos y libertades proclamados en esta Declaración, sin distinción alguna de raza, color, sexo, idioma, religión, opinión política o de cualquier otra índole, origen nacional o social, posición económica, nacimiento o cualquier otra condición. Además, no 
se hará distinción alguna fundada en la condición política, jurídica o internacional del país o territorio de cuya jurisdicción dependa una persona, tanto si se trata de un país independiente, como de un territorio bajo administración fiduciaria, no autónomo o sometido a cualquier otra limitación de soberanía.

La mayoría de quienes aceptan que existen esos derechos y con carácter universal, es decir, que todos los seres humanos son titulares de ellos, sostienen que son previos o, en todo caso, independientes de lo que puedan disponer las leyes, de lo que disponga el Derecho que, precisamente por esa razón (dispuesto por las leyes) llamamos <positivo $>$. Pero no hay acuerdo sobre su fundamento. Durante siglos se les calificó como "derechos naturales», es decir, previos a las leyes e independientes de ellas porque se encuentran implícitos en la naturaleza humana. Esa es la concepción iusnaturalista de los derechos humanos que, a su vez, ha recibido a lo largo de la historia diferentes formulaciones. Así, hay quienes creen en un orden divino (sobre natural), un plan impuesto por Dios o por los dioses a la naturaleza, que incluye una suerte de leyes no escritas que todo ser humano puede conocer, como apreciamos en el precioso diálogo que nos legó Sófocles, el famoso alegato de Antígona frente al tirano Creonte, que había decretado que Polinices, el hermano rebelde de Antígona, no debía recibir sepultura, mandato que Antígona desobedece porque, en su opinión, contradice leyes superiores y no escritas. Es la reivindicación de la igualdad biológica y cultural de todos los seres humanos que esgrime el judío Shylock, en su defensa ante Portia, en la obra de Shakespeare El Mercader de Venecia. De ese modo se desarrolla la doctrina del Derecho natural que sostiene que las leyes humanas (el Derecho positivo) que no son acordes con esas normas naturales, no escritas, no solo no son justas, sino que ni siquiera son Derecho. A partir de la Modernidad, estos derechos naturales son presentados más bien como expresión de un Derecho natural, sí, pero no necesariamente de fundamento religioso, sino asentado en las exigencias que la razón encuentra en la naturaleza humana. La universalidad de esos derechos, como la de esas leyes naturales, queda así asegurada por encima de las diferentes concepciones religiosas, porque la razón es, obviamente, universal. Desde esa concepción, que supone un cierto objetivismo ético (y que admitirá muchos matices), todos los seres humanos podemos conocer y reivindicar nuestros derechos. Esta es la clave, que entroncará con quienes, mucho tiempo después, invoquen los derechos desde otra concepción que no es necesariamente iusnaturalista, como la de Jhering, o en clave de satisfacción de las necesidades humanas básicas, quizá el criterio de fundamentación de los derechos humanos que tiene hoy mayor aceptación, también a la hora de establecer un catálogo y jerarquía de los mismos. Son las que se expresan por ejemplo en la moderna noción de seguridad humana en la que se basa a su vez el Indice de desarrollo bumano que utiliza Naciones Unidas y que, en realidad, constituye una manera de concretar la Declaración universal de los derechos humanos de 1948 y los Pactos de derechos humanos de 1966. 
JAVIER DE LUCAS

ALGUNOS ELEMENTOS BÁSICOS DE LA «CULTURA DE LOS DERECHOS» ¿POR QUÉ LOS DERECHOS HUMANOS?

Pero la importancia del iusnaturalismo racionalista radica sobre todo en que ofreció una palanca para limitar el hasta entonces poder absoluto de los reyes. En efecto, la apelación a derechos de todos los seres humanos, anteriores y jerárquicamente superiores a todo mandato del poder, aparece a la vez como límite y justificación de la obediencia a las leyes positivas. Con distintos acentos -los que separan las tesis de Hobbes de las de Locke- irá configurándose la idea de que cabe reivindicar derechos anteriores y superiores que pueden incluso justificar la rebelión frente al monarca que no los reconoce ni garantiza, una tesis que, como hemos visto ya se sostuvo en la antigua Grecia, pasando por las concepciones medievales de los denominados monarcómacos, que preconizan como justo el tiranicidio, hasta que Locke formulará con mayor precisión esa función política de los derechos, como barrera frente al poder, pero también como fundamento o criterio de juicio de su legitimidad, que inspirará el derecho de resistencia -Locke lo denominará un appeal to heaven, según escribe en el famoso capítulo 14 de su Segundo Tratado sobre el Gobierno civil (1689-90)-, verdadero motor de las revoluciones americana y francesa, las que conducirán a las primeras proclamaciones de esos derechos.

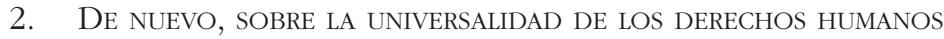

Por supuesto, la universalidad de los derechos humanos es un requisito conceptual -si no son universales, no pueden ser humanos- que debe ser entendido como una aspiración, una exigencia ("universabilidad»), una idea regulativa en el sentido kantiano, si no se quiere sucumbir a la crítica que nos presenta el desmentido empírico, esto es, la evidencia de que su reconocimiento y garantía efectivas para todos los seres humanos está desmentida por la realidad inmediata. Incluso hoy, ya casi en la segunda década del siglo xxi, más de doscientos cincuenta años después de esas primeras declaraciones de derechos de las revoluciones americana y francesa, para buena parte de la humanidad los derechos humanos siguen siendo buenas palabras. Pero la crítica inspirada en el pragmatismo no nos debe confundir. Retomemos a Jhering y reconozcamos cómo la exigencia de universalidad se ha abierto camino, al modo que una piedra lanzada al agua abre progresivos círculos concéntricos. Sí: esos derechos fueron durante mucho tiempo un privilegio real al alcance de unos pocos. Dependieron de circunstancias como el sexo, la edad, la clase socioeconómica, los rasgos etnoculturales, el lugar de nacimiento, la nacionalidad... y cada una de esas barreras ha ido cayendo gracias en buena medida a procesos históricos de lucha social, incluso de guerras, que acabaron (o al menos redujeron muy considerablemente) con la esclavitud, con el colonialismo, con el racismo, con la brutal concepción patriarcal. Sin duda, hoy persisten esas restricciones. Incluso reaparecen aquellas que creímos haber superado. Baste pensar en la reaparición hoy de formas de esclavitud respecto a inmigrantes, mujeres y niños sometidos a trabajos en condiciones inhumanas. O en el resurgimiento de movimientos que expresan el racismo o la xenofobia y que parecen recuperar la 
fábula que nos explicara Orwell en su Rebelión en la granja, ese sofisma según el cual todos los seres humanos seríamos iguales, pero algunos más iguales que otros.

Universalidad quiere decir igualdad o, si se prefiere la fórmula de Balibar, egalibertad $^{1}$ : todos los derechos, para todos los seres humanos, a fin de llegar a un modelo de democracia equitativa, inclusiva, plural, que no puede basarse en criterios de exclusión o discriminación supuestamente justificados por la nacionalidad, por ejemplo. La lucha por los derechos, a la que me he referido como motor y elemento básico de una cultura de los derechos, significa, en clave de universalidad, que ningún derecho de ningún ser humano puede serme ajeno. No podemos aceptar que esa lucha sea solo, pues, por nuestros derechos y por los derechos de los nuestros, si no es también y al mismo tiempo lucha por los derechos de los otros. Por una razón obvia: los derechos de los otros son los nuestros. Y eso no significa homogeneidad: cada uno de nosotros es diferente precisamente porque la característica reivindicada por la Ilustración como definitoria, la autonomía clave de la dignidad- significa ante todo libertad, fundamento de los derechos. La universalidad, por tanto, debe permitir que la igualdad sea entendida desde la diferencia y no pese o contra ella.

Podríamos decir que la lucha por la dignidad tiene dos ejes. De una parte, el eje que afecta a quienes pertenecen a la inmensa mayoría, a la mal llamada "normalidad", que no se identifica con una diferencia específica que les singularice, más allá, obviamente, de la propia individualidad. De otro, una lucha mucho más ardua, la que tiene como eje el reconocimiento de la dignidad de quienes han de conseguir construirla y convencer de ello a la mayoría desde su especificidad, desde su diferencia que, las más de las veces, es la coartada que sirve a la mayoría para rechazar, discriminar o expulsar a quienes reivindican esa condición diferencial. Llegar a visibilizarla, lo sabemos, cuesta ya un sacrificio muchas veces heroico que, además, debe rechazar la limosna, el paternalismo, tanto como la estigmatización y aun criminalización que en definitiva suponen la exclusión del espacio público, su reconocimiento como menos iguales, como menos seres humanos. Lo cierto es que la experiencia histórica nos enseña que las mayorías son cambiantes, que el nosotros puede desaparecer y ser sustituido. Y nos enseña también que llegar a formar parte de esa condición de igual-libertad de la que disfrutan los miembros de la mayoría dominantes implica un proceso de luchas sociales (los protagonizados por las mujeres, los esclavos, los obreros, los colonizados...) muchas veces prolongado y difícil. En definitiva, cada uno de nosotros -lo enseñaron San Agustín, Rimbaud o Pessoa- es también otro y por eso luchando por sus derechos luchamos por los míos. Es decir, la lucha por los derechos es también una tarea de solidaridad. Y así vemos que este programa es el viejo programa revolucionario: libertad, igualdad, fraternidad.

1. Cfr. la edición en castellano de sus escritos en torno a esta propuesta, La igualibertad. Barcelona: Herder, 2017. 

¿POR QUÉ LOS DERECHOS HUMANOS?

A mi juicio, bien podríamos decir que las lecciones elementales, las consideraciones mínimas que podemos deducir del sentido común y de la experiencia histórica sobre la universalidad de los derechos humanos son, en realidad, tres.

La primera, que su contenido concreto, su expresión más inmediata, es la igualdad. Por eso la lucha contra el negativo de la igualdad, contra las manifestaciones injustas, inaceptables de desigualdad que son las expresiones de la discriminación, la lucha contra toda forma de discriminación (empezando por la más inaceptable, la discriminación de derechos que se basa en la distinción de género, entre hombres y mujeres, como entendió bien la Organización de las Naciones Unidas, cuya primera Convención se dirige precisamente a este propósito), es la primera exigencia derivada de la concepción universal de los derechos humanos. Aquí se abre una discusión que debe ser analizada sosegada y racionalmente: lo que encontramos en la naturaleza, en los hechos, no es desigualdad, sino diferencia. La desigualdad no es natural, sino una respuesta normativa, una forma de tratar la realidad, que nos muestra por doquier diversidad, diferencia. El que postulemos que el trato que se debe dar es el de igualdad responde a razones que, como advirtiera Aristóteles, en casos concretos pueden justificar un trato discriminatorio, discriminaciones que consideraremos justas, con arreglo a razones. Pero, en principio, lo que nos enseña la experiencia histórica es que la igualdad es la respuesta más justa y que quien reivindique o imponga desigualdad debe cargar con el peso de justificar esa excepción.

La segunda lección es que esta es una historia inacabada, inacabable: lo es porque incluso si conseguimos garantizar la igualdad frente a las condiciones «naturales» en las que se ha basado la discriminación (el sexo/género, la edad, el nacimiento, etc.), surgen nuevas condiciones culturales que pueden propiciar discriminaciones injustas, sobre todo a caballo de las innovaciones tecnológicas. Esta lucha por la igualdad encuentra nuevos frentes. Y, además, como nos muestra la historia y como desgraciadamente constatamos hoy, nada se puede dar por adquirido, ni siquiera la victoria sobre el machismo, el racismo o la xenofobia: la eliminación de las formas de discriminación exige una atención constante y es tarea de todos, esfuerzo permanente que a todos nos compete. Empezando por el igual respeto a la vida de todos los seres humanos: todas las vidas humanas importan, deben importar igual; no solo las de quienes son como nosotros, las de los nuestros. De ahí que la lucha por el igual respeto a los derechos de quienes son diferentes es un buen criterio para juzgar el avance de la universalidad y que podamos asegurar por ejemplo que la lucha por los derechos de las mujeres, de los homosexuales, de los discapacitados, como la lucha por la salud y contra el hambre que padecen los sudaneses del sur, o contra la discriminación que sufren los robyingas, es la lucha por nuestros derechos. Esto quiere decir, además, que la universalidad es también unidad de los derechos, de todos los derechos de todos los seres bumanos. No solo de las libertades públicas, sino también de esos derechos económicos, sociales y culturales que son la piedra de toque de la igualdad. Porque si algo significa la universalidad de los derechos es lo que expresa el término propuesto por Etiénne 
Balibar: egalibertad. No hay universalidad si no se dan garantías para el acceso igual a los derechos que satisfacen necesidades básicas: salud, educación, trabajo, vivienda. Y eso obliga en primer lugar a ocuparse de quienes, por condiciones no elegidas, padecen a ese respecto de una desigualdad incluso heredada y transmitida. Los derechos humanos no son solo el fundamento de legitimidad del orden social, sino también y sobre todo el ideal regulativo, la concreción histórica de la idea de justicia a la que debemos aspirar aun conscientes de que su plenitud está fuera del alcance de la dimensión legal, de la acción política.

\section{LOS DERECHOS HUMANOS, CONDICIÓN DE LEGITIMIDAD}

Precisamente la tercera lección elemental es que esta lucha exige medios y que estos a su vez precisan de la voluntad política. La universalidad de los derechos depende sobre todo del compromiso de los Estados por asumir la responsabilidad que les toca en el reconocimiento y garantía efectiva de los derechos de todos los seres humanos: de todos, no solo de sus ciudadanos, sus primeros acreedores, sino también de cuantos transitan o residen en su territorio sin esa condición de ciudadanía plena. Y asimismo finalmente, en buena medida, aunque esto pueda parecer una obligación supererogatoria, de los derechos de todos los seres humanos, porque vivimos en una condición global que obliga a replantear la noción de soberanía, sus atributos y límites, su legitimidad. Un eminente jurista italiano, Luigi Ferrajoli, ha sabido enunciar esa transformación epocal a la que nos enfrentamos y, a mi juicio, mejor que muchos otros que comparten la misma tesis: escribe Ferrajoli que la soberanía en su concepción estatal-nacional al modo en que la concibieron Hobbes y Bodin se enfrenta un oxímoron cuando se trata de cohonestarla con el desarrollo coherente de las exigencias del Estado de Derecho y aun de la democracia, en un mundo que ya no es el del orden de Westfalia, sino la aldea global. El test de esa contradicción irresoluble es precisamente la necesidad de garantizar de modo efectivo los derechos humanos entendidos como universales, que exigen una reformulación del principio de soberanía al mismo tiempo que suponen una fuerte exigencia para los Estados. Y ello sin ignorar la novedad que supone la irrupción de una generalizada toma de conciencia de la responsabilidad, que nos interpela a todos respecto a su protección y que ha dado lugar al creciente activismo de los movimientos sociales, de las organizaciones transnacionales (las ONG), y que nace de la toma de conciencia de nuestra propia responsabilidad individual, la de cada uno de nosotros, a ese respecto.

Este es un cambio en la conciencia de la humanidad, de todos y cada uno de nosotros. La aldea global ha puesto ante nuestros ojos lo inaceptable radical, esto es, el daño que suponen las violaciones de los derechos humanos que padecen millones de personas en todo el mundo. Ese daño, ese mal inaceptable que afecta a tantos otros, nos interpela a cada uno de nosotros. Por eso, los derechos humanos exigen voluntad política de compromiso. Sin esa voluntad política, concretada en medios humanos y materiales y sobre todo, en instrumentos independientes de 
control, no solo sociales sino también, sí, institucionales, los derechos no son más que papel mojado.

Por eso también, esta es, insisto, la tercera lección, los derechos humanos son siempre una cuestión de política. Pero son ante todo la cuestión política: están en el centro del vínculo social y político y, por supuesto, en el centro de la democracia. En el sentido radical, es decir, profundo, son la cuestión política por excelencia. Por eso hoy es inimaginable una propuesta política, un proyecto de sociedad, que no los tenga en su centro y nuestra tarea, como ciudadanos, es exigir que así sea. De nuevo llegamos a la idea de la que partí: la lucha por los derechos como una tarea ciudadana, que postula a su vez un modelo de ciudadanía activa, participativa, y no solo -que es importantísimo- desde el punto de vista del control de las acciones de los poderes que deben garantizar y desarrollar los derechos (por tanto, a fortiori, de las acciones que puedan ponerlos en riesgo), sino también constructiva, propositiva. 